\title{
Enucleación secundaria a descompresión o marsupialización para pacientes con queratoquiste.
}

\section{Secondary enucleation to decompression/marsupialization for keratocystic odontogenic tumors.}

\author{
Sofía Pardo ${ }^{1,2}$, Francisca Retamal ${ }^{1,2}$, Luis Romo ${ }^{1,2,3^{*}}$
}

\begin{abstract}
1. Escuela de Odontología, Facultad de Medicina, Pontificia Universidad Católica de Chile, Santiago, Chile

2. Proyecto Epistemonikos, Santiago, Chile

3. Central Odontológica, Servicio de Cirugía y

Traumatología Maxilofacial, Hospital Clínico Fuerza

Aérea de Chile, Santiago, Chile
\end{abstract}

* Correspondencia Autor: Luis Romo | Dirección: Centro Evidencia UC, Pontificia Universidad

Católica de Chile, Diagonal Paraguay 476,

Santiago, Chile | E-mail: Iromos@uc.cl

\section{RESUMEN}

Introducción: El queratoquiste o tumor odontogénico queratoquístico es una de las neoplasias odontogénicas "benignas" más frecuentes. Existen múltiples opciones de tratamiento, pero no existe consenso sobre ellas. Este resumen busca evaluar la efectividad de la enucleación secundaria a descompresión o marsupialización. Métodos: Realizamos una búsqueda en Epistemonikos, la mayor base de datos de revisiones sistemáticas en salud, la cual es mantenida mediante el cribado de múltiples fuentes de información, incluyendo MEDLINE, EMBASE, Cochrane, entre otras. Extrajimos los datos desde las revisiones identificadas, analizamos los datos de los estudios primarios, realizamos un metaanálisis y preparamos una tabla de resumen de los resultados utilizando el método GRADE. Resultados y conclusiones: Identificamos cuatro revisiones sistemáticas que en conjunto incluyeron cinco estudios primarios, los cuales corresponden a estudios observacionales. Concluimos que no es posible establecer con claridad si la enucleación secundaria a descompresión o marsupialización disminuye la recidiva de queratoquiste. Otros desenlaces como dolor postoperatorio, infección y fractura patológica no fueron reportados.

\section{ABSTRACT}

Introduction: Keratocystic odontogenic tumor is one of the most common odontogenic neoplasms. Many treatment modalities have been recommended for the treatment of keratocystic, but there is no consensus regarding the optimal treatment. This summary seeks to evaluate the effectiveness of enucleation secondary to decompression or marsupialization. Methods: We searched in Epistemonikos, the largest database of systematic reviews in health, which is maintained by screening multiple information sources, including MEDLINE, EMBASE, Cochrane, among others. We extracted data from the systematic reviews, reanalyzed data of primary studies, conducted a metaanalysis and generated a summary of findings table using the GRADE approach. Results and conclusions: We identified 4 systematic reviews including 5 studies overall, all corresponding to observational studies. There is uncertainty whether secondary enucleation to decompression/marsupialization reduces recurrence rate as the certainty of the evidence has been assessed as very low. No studies were found that looked at postoperative pain, infection and pathologic fracture.

\section{KEY WORDS}

Keratocyst; Decompression; Marsupialization; Enucleation; Epistemonikos; GRADE.

Int. J. Inter. Dent Vol. 13(3); 224-228, 2020. 


\section{PROBLEMA}

El queratoquiste o tumor odontogénico queratoquístico es una de las lesiones odontogénicas benignas más frecuentes, representa un 12$14 \%$ de los quistes odontogénicos ${ }^{[1]}$. Se caracteriza por ser agresivo, de crecimiento expansivo y altamente recurrente en alguna de sus variedades. Existen numerosas alternativas de tratamiento para el manejo de estas lesiones como descompresión, marsupialización, enucleación, diferentes medidas adyuvantes y finalmente la resección ósea ${ }^{[2]}$, pero la elección sigue siendo controvertida.

Dentro de las alternativas de tratamiento más conservadoras, se encuentran la descompresión y marsupialización, en las cuales se realiza una pequeña incisión y se utiliza una cánula de drenaje que reduce la presión en la lesión. Sin embargo, su uso como tratamiento definitivo depende de factores propios del paciente, como una condición sistémica que lo haga más propenso a infecciones secundarias a la intervención, consumo de medicamentos o por la disposición del paciente a usar la cánula, y del tamaño de la lesión ${ }^{[1]}$.

Debido a lo anterior, se ha propuesto el uso de la enucleación, que se realiza de forma secundaria a lo anterior y que consiste en la extirpación de la lesión a través de una intervención quirúrgica. El uso complementario de estas terapias aseguraría la remoción completa de la lesión, al disminuir el tamaño del quiste y permitir una extirpación más fácil, disminuyendo su tasa de recurrencia ${ }^{[1]}$.

Este resumen tiene como objetivo evaluar la efectividad y seguridad de la adición de la enucleación a la descompresión o marsupialización.

\section{MÉTODOS}

Realizamos una búsqueda en Epistemonikos, la mayor base de datos de revisiones sistemáticas en salud, la cual es mantenida mediante búsquedas en múltiples fuentes de información, incluyendo MEDLINE, EMBASE, Cochrane, entre otras. Extrajimos los datos desde las revisiones identificadas y analizamos los datos de los estudios primarios. Con esta información, generamos un resumen estructurado denominado FRISBEE (Friendly Summaries of Body of Evidence using Epistemonikos), siguiendo un formato preestablecido, que incluye mensajes clave, un resumen del conjunto de evidencia (presentado como matriz de evidencia en Epistemonikos), metanálisis del total de los estudios cuando sea posible, una tabla de resumen de resultados con el método GRADE y una sección de otras consideraciones para la toma de decisión

\section{Mensajes clave}

- No está claro si una enucleación secundaria a descompresión o marsupialización disminuye la recidiva de queratoquiste, debido a que la certeza de la evidencia existente ha sido evaluada como muy baja.

- No se encontraron estudios que evaluaran el dolor postoperatorio, riesgo de infección o fractura patológica asociado al tratamiento con enucleación secundaria a descompresión o marsupialización.

\section{Acerca del conjunto de evidencia para esta pregunta}

Cuál es la evidencia

Véase matriz de evidencia en Epistemonikos más abajo.

Encontramos cuatro revisiones sistemáticas ${ }^{[1],[3], ~[4], ~[5] ~ q u e ~ i n c l u y e r o n ~}$ cinco estudios primarios ${ }^{[6],[7],[8],[9],[10],}$ todos correspondientes a estudios observacionales.

Los estudios incluyeron pacientes jóvenes y adultos, de edades entre 15 a 68 años ${ }^{[7]}, 18$ a 90 años ${ }^{[8]}, 16$ a 31 $a^{n} 0 s^{[9]}$ y 13 a 70 años $^{[10]}$. El promedio de edad de los participantes de un estudio ${ }^{[6]}$ no fue reportado.

Cuatro estudios incluyeron pacientes no sindrómicos con diagnóstico de queratoquiste y confirmación histopatológica ${ }^{[7],[8],[9],[10]}$ y un estudio ${ }^{[6]}$ incluyó pacientes sindrómicos

Qué tipo de pacientes incluyeron los estudios* Gorlin).

Cuatro estudios incluyeron pacientes con queratoquiste único ubicado en el maxilar o en la mandíbula[7], [8], [9], [10]. Sin embargo, el $95 \%$ de los pacientes que se reportan en los estudios tenían queratoquiste en la mandíbula específicamente en la región posterior.

El $60 \%$ de los pacientes participantes en los estudios fueron de sexo masculino ${ }^{[6], ~[7], ~[8], ~[9], ~[10] . ~}$

Todos los estudios compararon enucleación secundaria a descompresión o marsupialización contra descompresión o marsupialización solas.

Un estudio ${ }^{[8]}$ comparó enucleación secundaria a descompresión contra descompresión y enucleación, por

Qué tipo de intervenciones separado.

incluyeron los estudios*

Dos estudios ${ }^{[9]}$ y ${ }^{[10]}$ evaluaron enucleación secundaria a marsupialización contra marsupialización.

Un estudio ${ }^{[7]}$ evaluó enucleación secundaria a descompresión, enucleación secundaria a marsupialización y descompresión y marsupialización por separado.

Las revisiones sistemáticas reportaron un único desenlace:

Qué tipo de desenlaces midieron

- Recidiva de la lesión.

El seguimiento promedio de los estudios fue de 89 meses $(7,42$ años) con un rango que fluctuó entre 9 y 118 meses (9,8 años).

* La información sobre los estudios primarios es extraída desde las revisiones sistemáticas identificadas, no directamente desde los estudios, a menos que se especifique lo contrario.

\section{RESUMEN DE LOS RESULTADOS}

La información sobre los efectos de enucleación secundaria a descompresión o marsupialización en pacientes con queratoquiste está basada en 5 estudios observacionales que incluyeron 78 pacientes.

Todos los estudios midieron el desenlace recidiva (78 pacientes) ${ }^{[6]}$, [7], [8], [9], [10]. Los desenlaces fractura patológica, dolor postoperatorio e infección no fueron reportados por la evidencia analizada.

El resumen de los resultados es el siguiente:

-No es posible establecer con claridad si la enucleación secundaria a descompresión aumenta/disminuye la recidiva de queratoquiste, debido a que la certeza de la evidencia existente ha sido evaluada como muy baja.

-No se encontraron estudios que evaluaran fractura mandibular 
como consecuencia del tratamiento con enucleación secundaria a descompresión o marsupialización.

- No se encontraron estudios que evaluaran el desarrollo de infección asociada al tratamiento con enucleación secundaria a descompresión o marsupialización.

- No se encontraron estudios que evaluaran el dolor postoperatorio asociado al tratamiento con enucleación secundaria a descompresión o marsupialización.

\section{Enucleación secundaria a descompresión o marsupialización en pacientes con queratoquiste}

\section{Pacientes Intervención Comparación}

Pacientes con queratoquiste

Descompresión o marsupialización más enucleación

\begin{tabular}{|l|}
\hline \multirow{2}{*}{ Desenlaces } \\
\cline { 2 - 2 }
\end{tabular}

Descompresión o marsupialización

\begin{tabular}{|c|c|c|c|c|}
\hline \multirow[b]{2}{*}{ Recidiva } & 207 por 1000 & 74 por 1000 & \multirow{2}{*}{$\begin{array}{l}\text { RR } 0,36 \\
(0,10 \text { a } \\
1,30)\end{array}$} & \multirow{2}{*}{$\begin{array}{l}\oplus \bigcirc \bigcirc \bigcirc^{1,2} \\
\text { Muy baja }\end{array}$} \\
\hline & \multicolumn{2}{|c|}{$\begin{array}{l}\text { Diferencia: } 133 \text { pacientes menos } \\
\text { (Margen de error: } 186 \text { menos a } 62 \text { más) }\end{array}$} & & \\
\hline $\begin{array}{l}\text { Fractura } \\
\text { patológica }\end{array}$ & \multicolumn{3}{|c|}{$\begin{array}{c}\text { El desenlace fractura patológica no fue medido o reportado } \\
\text { por los estudios. }\end{array}$} & -- \\
\hline Infección & \multicolumn{3}{|c|}{$\begin{array}{c}\text { El desenlace infección no fue medido o reportado por los } \\
\text { estudios. }\end{array}$} & -- \\
\hline $\begin{array}{l}\text { Dolor } \\
\text { postoperatorio }\end{array}$ & \multicolumn{3}{|c|}{$\begin{array}{l}\text { El desenlace dolor postoperatorio no fue reportado o } \\
\text { medido por los estudios }\end{array}$} & \\
\hline
\end{tabular}

Margen de error: Intervalo de confianza del 95\% (IC 95\%).

RR: Riesgo relativo

GRADE: Grados de evidencia del GRADE Working Group (ver más adelante).

* Los riesgos SIN enucleación están basados en los riesgos del grupo control en los estudios. El riesgo CON enucleación (y su margen de error) está calculado a partir del efecto relativo (y su margen de error).

${ }^{1}$ Estudios observacionales.

${ }^{2}$ Se disminuyó un nivel de certeza de evidencia por imprecisión, ya que las decisiones a ambos extremos del intervalo de confianza podrían variar.

\begin{tabular}{|l|}
\hline Acerca de la certeza de la evidencia (GRADE)* \\
\hline$\oplus \oplus \oplus \oplus$ \\
Alta: La investigación entrega una muy buena indicación del efecto probable. La probabilidad de \\
que el efecto sea sustancialmente distinto† es baja. \\
$\oplus \oplus \oplus \bigcirc$ \\
Moderada: La investigación entrega una buena indicación del efecto probable. La probabilidad de \\
que el efecto sea sustancialmente distinto† es moderada. \\
$\oplus \oplus \bigcirc \bigcirc$ \\
Baja: La investigación entrega alguna indicación del efecto probable. Sin embargo, la probabilidad \\
de que el efecto sea sustancialmente distinto† es alta. \\
$\oplus \bigcirc \bigcirc \bigcirc$ \\
Muy baja: La investigación no entrega una estimación confiable del efecto probable. La \\
probabilidad de que el efecto sea sustancialmente distinto† es muy alta. \\
\hline *Esto es también denominado 'calidad de la evidencia' o 'confianza en los estimadores del efecto'. \\
$\dagger$ Sustancialmente distinto = una diferencia suficientemente grande como para afectar la decisión \\
\hline
\end{tabular}




\section{OTRAS CONSIDERACIONES PARA LA TOMA DE DECISIÓN}

\section{A quién se aplica y a quién no se aplica esta evidencia}

- La evidencia presentada en este resumen es aplicable a adolescentes y adultos con diagnóstico clínico y confirmación histopatológica de queratoquiste. Sin distinción de género.

- Pese a que la mayoría de los pacientes incluidos en los estudios presentaban lesiones en la mandíbula, es razonable extrapolar estos resultados a pacientes con lesiones de queratoquistes en el maxilar.

- Los resultados no son extrapolables a pacientes con lesiones multiloculares.

\section{Sobre los desenlaces incluidos en este resumen}

- Los desenlaces seleccionados son aquellos considerados críticos para la toma de decisión de acuerdo a la opinión de los autores de este resumen. Sin embargo, sólo el desenlace recidiva fue reportado por las revisiones sistemáticas identificadas.

\section{Balance daño/beneficio y certeza de la evidencia}

- No existe claridad respecto a la efectividad o seguridad de la enucleación secundaria a descompresión o marsupialización como tratamiento de queratoquiste, porque la certeza de la evidencia es muy baja. Adicionalmente, no existe evidencia respecto al efecto de la intervención sobre dolor postoperatorio, infección o fracturas patológicas de los pacientes con queratoquiste, ya que estos desenlaces no fueron presentados en la evidencia analizada.

- En consideración de lo anterior, no es posible realizar un adecuado balance entre beneficios y riesgos y se considera que la decisión debería evaluarse de manera individualizada, teniendo en cuenta factores adicionales asociados a la experiencia y preferencias tanto de los pacientes como tratantes.

\section{Consideraciones de recursos}

- Ninguna revisión sistemática se refirió a los recursos o costos involucrados en las opciones de tratamiento.

- La adición de la enucleación al tratamiento con descompresión implica un mayor costo para el paciente porque involucra una sesión quirúrgica adicional. Sin embargo, aun cuando se observa una posible disminución de recidiva en pacientes que fueron tratados con enucleación secundaria a descompresión o marsupialización, no corresponde hacer un análisis de costo-efectividad hasta que se pruebe su beneficio y se evalúen sus riesgos.

\section{Qué piensan los pacientes y sus tratantes}

- Enfrentados a la evidencia presentada en este resumen, la mayoría de los pacientes y tratantes deberían inclinarse en contra de la intervención. Sin embargo, dependiendo de la experiencia positiva de los tratantes con la enucleación secundaria, algunos podrían preferir su uso, ya que con una lesión considerablemente más pequeña resultaría más fácil su extirpación quirúrgica. Debido a que la técnica con una previa descompresión, reduce los niveles de IL-1a y citoqueratina-10, responsables del aumento del quiste ${ }^{[5]}$.

- Es importante destacar que el éxito de la descompresión requiere de un rol activo y responsable del paciente, ya que esta técnica considera la instalación de una cánula de drenaje que debe permanecer en la cavidad bucal del paciente por lo menos tres a cuatro meses, siguiendo rigurosamente las indicaciones de irrigación diaria. La incomodidad y constancia asociada al tratamiento deben ser factores a considerar de manera individualizada en la toma de decisión.

\section{Diferencias entre este resumen y otras fuentes}

- Las conclusiones de este resumen difieren con las revisiones sistemáticas seleccionadas, ya que las revisiones concluyen que la enucleación secundaria a descompresión minimiza la recidiva. Estas diferencias pueden deberse a que ninguna de las revisiones evaluó la certeza de la evidencia de los desenlaces.

- Además en algunas revisiones ${ }^{[2],[3]}$ se considera importante utilizar terapias adyuvantes como la solución de Carnoy, para reducir la recidiva.

- El protocolo propuesto por la sociedad española de cirugía maxilofacial recomienda tratamientos más radicales, como enucleación de la lesión con raspado óseo periférico y escisión de la mucosa que lo recubre. Además recomiendan la cauterización química posterior con solución de Carnoy o nitrógeno líquido. Y en casos excepcionales, puede ser necesaria la resección parcial ósea con reconstrucción del defecto, preferiblemente de forma inmediata ${ }^{[11]}$.

\section{¿Puede que cambie esta información en el futuro?}

- Es muy probable que las conclusiones de este resumen cambien a futuro frente a nueva evidencia, debido a la incertidumbre existente.

- No se identificaron ensayos aleatorizados en curso en International Clinical Trials Registry Platform de la Organización Mundial de la Salud

- No se identificaron revisiones sistemáticas en curso en plataforma PROSPERO.

\section{CÓMO REALIZAMOS ESTE RESUMEN}

Mediante métodos automatizados y colaborativos recopilamos toda la evidencia relevante para la pregunta de interés y la presentamos en una matriz de evidencia.

Siga el enlace para acceder a la versión interactiva: Enucleación secundaria a descompresión o marsupialización comparado con descompresión o marsupialización para pacientes con queratoquiste

\section{NOTAS}

Si con posterioridad a la publicación de este resumen se publican nuevas revisiones sistemáticas sobre este tema, en la parte superior de la matriz se mostrará un aviso de "nueva evidencia".

Este artículo es parte del proyecto síntesis de evidencia de Epistemonikos. Se elabora con una metodología preestablecida, siguiendo rigurosos estándares metodológicos y proceso de revisión por pares interno. Cada uno de estos artículos corresponde a un resumen, denominado FRISBEE (Friendly Summary of Body of Evidence using Epistemonikos), cuyo principal objetivo es sintetizar el conjunto de evidencia de una pregunta específica, en un formato amigable a los profesionales clínicos. Sus principales recursos se basan en la matriz de evidencia de Epistemonikos y análisis de resultados usando metodología GRADE. Mayores detalles de los métodos para elaborar este FRISBEE están descritos aquí (http://dx.doi.org/10.5867/medwave.2014.06.5997)

La Fundación Epistemonikos es una organización que busca acercar la información a quienes toman decisiones en salud, mediante el uso de tecnologías. Su principal desarrollo es la base de datos Epistemonikos (www.epistemonikos.org).

\section{DECLARACIÓN DE CONFLICTOS DE INTERESES}

Los autores declaran no tener conflictos de intereses con la materia de este artículo.

\section{AGRADECIMIENTOS}

Este resumen de evidencia fue elaborado con el apoyo metodológico del Centro Evidencia UC, Facultad de Medicina, Pontificia Universidad Católica de Chile. 


\section{Bibliografía}

1. Al-Moraissi EA, Pogrel MA, Ellis E 3rd. Enucleation with or without adjuvant therapy versus marsupialization with or without secondary enucleation in the treatment of keratocystic odontogenic tumors: A systematic review and meta- analysis. J Craniomaxillofac Surg. 2016 Sep;44(9):1395-403. doi:10.1016/j.jcms.2016.05.020. Epub 2016 May 31. PMID: 27452613.

2. Slusarenko da Silva, Y., Stoelinga, P.J.W. \& Naclério-Homem, M.d. Recurrence of nonsyndromic odontogenic keratocyst after marsupialization and delayed enucleation vs. enucleation alone: a systematic review and meta-analysis. Oral Maxillofac Surg 23, 1-11 (2019). https://doi.org/10.1007/s10006-018-0737-3

3. Al-Moraissi EA, Dahan AA, Alwadeai MS, Oginni FO, Al-Jamali JM, Alkhutari AS, Al-Tairi NH, Almaweri AA, Al-Sanabani JS. What surgical treatment has the lowest recurrence rate following the management of keratocystic odontogenic tumor?: A large systematic review and meta-analysis. J Craniomaxillofac Surg. 2017 Jan;45(1):131-144. doi: 10.1016/j.jcms.2016.10.013. Epub 2016 Oct 31. PMID: 27955959.

4. Wushou A, Zhao YJ, Shao ZM. Marsupialization is the optimal treatment approach for keratocystic odontogenic tumour. J Craniomaxillofac Surg. 2014 Oct;42(7):15404. doi: 10.1016/j.jcms.2014.04.027. Epub 2014 May 2. PMID: 24993466.

5. de Castro MS, Caixeta CA, de Carli ML, Ribeiro Júnior NV, Miyazawa M, Pereira AAC, Sperandio FF, Hanemann JAC. Conservative surgical treatments for nonsyndromic odontogenic keratocysts: a systematic review and meta-analysis. Clin Oral Investig. 2018 Jun;22(5):2089-2101. doi: 10.1007/s00784-017-2315-8.
Epub 2017 Dec 20. PMID: 29264656.

6. Habibi A, Saghravanian N, Habibi M, Mellati E, Habibi M. Keratocystic odontogenic tumor: a 10-year retrospective study of 83 cases in an Iranian population. J Oral Sci. 2007 Sep;49(3):229-35. doi: 10.2334/josnusd.49.229. PMID: 17928730.

7. Awni S, Conn B. Decompression of keratocystic odontogenic tumors leading to increased fibrosis, but without any change in epithelial proliferation. Oral Surg Oral Med Oral Pathol Oral Radiol. 2017 Jun;123(6):634-644. doi: 10.1016/j. oooo.2016.12.007. Epub 2016 Dec 30. PMID: 28377093.

8. Kolokythas A, Fernandes RP, Pazoki A, Ord RA. Odontogenic keratocyst: todecompress or not to decompress? A comparative study of decompression and enucleation versus resection/peripheral ostectomy. J Oral Maxillofac Surg. 2007 Apr;65(4):640-4. doi: 10.1016/j.joms.2006.06.284. PMID: 17368357.

9. Tabrizi R, Özkan BT, Dehgani A, Langner NJ. Marsupialization as a treatment option for the odontogenic keratocyst. J Craniofac Surg. 2012 Sep;23(5):e459-61. doi: 10.1097/SCS.0b013e31825b3308. PMID: 22976707.

10. Nakamura N, Mitsuyasu T, Mitsuyasu Y, Taketomi T, Higuchi Y, Ohishi M. Marsupialization for odontogenic keratocysts: long-term follow-up analysis of the effects and changes in growth characteristics. Oral Surg Oral Med Oral Pathol Oral Radiol Endod. 2002 Nov;94(5):543-53. doi: 10.1067/moe.2002.128022. PMID: 12424446.

11. García L., Arroyo S., Bilbao A., Cebrian J. Protocolos clínicos de la Sociedad Española de Cirugía Oral y Maxilofacial. Capítulo 5: Quistes Maxilares. 2014 